Fatigue performance of distributed optical fiber sensors in reinforced concrete elements

\author{
António Barrias ${ }^{a *}$, Joan R. Casas ${ }^{\mathrm{a}}$ and Sergi Villalba ${ }^{\mathrm{b}}$ \\ ${ }^{a}$ Department of Civil and Environmental Engineering, Technical University of \\ Catalonia, UPC-BarcelonaTech, c/ Jordi Girona 1-3, 08034 Barcelona, Spain; \\ ${ }^{b}$ Department of Project and Construction Engineering, Technical University of \\ Catalonia, UPC-BarcelonaTech, c/ Colom 11, 08222 Terrassa, Spain
}

Corresponding author: Tel.: +34934016200 email: antonio.jose.de.sousa@upc.edu 


\section{Fatigue performance of distributed optical fiber sensors in reinforced concrete elements}

In this paper, the authors present the results of a laboratory test where two reinforced concrete beams were instrumented with Distributed Optical Fiber Sensors (DOFS) to allow the monitoring $g$ of strain in four different longitudinal segments bonded to their bottom surface. The test objective was to confirm the ability and good performance of the DOFS to monitor bridge structures in a longterm basis. To this end, the two specimens were submitted to a fatigue test up to 2 million load cycles. The amplitude of the stress range applied during the fatigue test was representative of what is expected on a standard highway bridge under vehicular traffic. Additionally, each of the four DOFS segments was bonded using a different adhesive to also investigate on the fatigue performance of the adhesive agents normally used. Finally, the collected data is checked against the data recorded with strain gauges also deployed on the beam specimens.

Keywords: Distributed optical fiber sensors, Rayleigh backscatter, structural health monitoring, sensor bonding, fatigue, cycle-loading

Subject classification codes: include these here if the journal requires them

\section{Introduction}

All civil engineering infrastructures are subjected to the passage of time and its associated decay as well as a number of different external adverse effects, which compromises their structural integrity, causes important economic losses, severe environmental impact and finally affects the safety of their users. As of 2016, in the United States alone, 39\% of the highway bridges in the National Bridge Inventory were older than 50 years and $9,1 \%$ of the total number of bridges were considered structurally deficient. As a result, an average of 188 million trips were performed daily across these structurally deficient bridges and the most recent estimate projects the backlog of rehabilitation projects for these infrastructures at $\$ 123$ billion [1]. 
It is therefore of paramount importance to properly monitor and maintain these infrastructures by the deployment of effective and adequate management strategies that optimize their use and extend their lifetime service. It is in this context that the field of Structural Health Monitoring (SHM) has been researched and developed during the past decades [2]. Nonetheless, SHM has not been practiced in a large scale and in a systematic manner quite yet in civil engineering structures, mostly due to the lack of reliable and affordable generic monitoring solutions [3].

Traditionally, monitoring practices have been conducted by means of visual inspections and through the use of a limited number of electric based sensors such as accelerometers, inclinometers, displacement transducers and strain sensors. All of these techniques present different challenges when applied in real world conditions [4].

It is in this way, that optical fiber sensors (OFS) have been one of the most popular research topics within SHM practices in the last two decades [5]. These type of sensors when compared with the conventionally used electrical sensors provide the enhanced advantages of being immune to electromagnetic interference, withstanding wide range of temperature variations, chemically inert and also being small and lightweight which facilitates its handling and transport [6], [7]. As of today, the most popular applications of this technology have been made through the use of discrete or quasi-distributed Fiber Bragg Grating (FBG) sensors [8].

Nevertheless, for a wide range of applications, especially when dealing with large-scale infrastructures, the number of point sensors that are required in order to obtain a complete strain information monitoring can increase dramatically. Additionally, for the specific case of concrete structures, where it is practically impossible to know beforehand with certainty the exact location of possible crack formations, these point sensors present obvious limitations. If the instrumented point 
sensors are not properly located, important data is going to be absent from the sensors' acquired data. Furthermore, looking into the logistics and more practical side of these applications, a large number of sensors also present the difficulty of requiring an associated large number of connecting cables making the full monitoring system more complex.

Distributed optical fiber sensors (DOFS) overcome this by providing distributed strain and temperature measurements with the unique advantage of doing so over the entire length of the optical fiber cable while sharing the same advantages of the other optical fiber sensors. In this way, virtually every cross-section of the structural element is being monitored while only requiring up to one single sensor and one corresponding connecting cable [3].

These sensors can be either bonded or embedded into the monitored structure and when strain or temperature variations occur, the scattered signal being reflected within the fiber cable is proportionally altered and in this way distributed sensing is achieved. This is the phenomenon behind this distributed optical fiber sensing as defined by the interaction between the emitted light and the physical optical medium. There are three types of scattering processes which allow for distributed sensing, namely Raman, Brillouin and Rayleigh scattering [9].

The Brillouin scattering based DOFS have been the most used and researched among the distributed sensors practiced in civil engineering SHM applications due to the large-range capability of several kilometers provided by these type of sensors [10], [11]. Nonetheless, as being deployed mostly using optical time domain reflectometry (OTDR), this scattering technique inherently provides a relatively low spatial resolution of $1 \mathrm{~m}$, which is not acceptable for numerous applications, including crack detection in reinforced concrete elements. 
On the other hand, Rayleigh scattering based DOFS, which uses optical frequency domain reflectometry (OFDR), enable an intrinsic higher spatial resolution, which can be as high as one millimetre being in this way suitable for damage detection and location [12]. Notwithstanding, it currently presents a significantly lower range of $70 \mathrm{~m}$ although this is expected to be greatly enhanced in the near future.

Consequently, this later is the scattering technique being researched by the authors in reinforced concrete elements SHM applications, through the use of an optical backscattered reflectometry (OBR) system based on the aforementioned Rayleigh OFDR. This technology is presented in greater detail in [9], [13].

\section{Fatigue load test: motivation}

Despite successful and promising applications where distributed optical fiber sensing technology based on Rayleigh scattering OFDR was used [14]-[18], due to its relatively novelty there are still several important challenges regarding its use in the monitoring of civil engineering infrastructures.

Several aspects still present some uncertainties and lack of knowledge that must be addressed before a more reliable use and wider application could be foreseen. This is, for instance, the case of the bonding techniques to different surfaces (concrete, steel) and the most appropriate bonding agents, where important developments were achieved recently [19]. However, a still open issue is the performance of these sensors regarding the accuracy and reliability of the measurements over time when monitoring real world structures during long-term periods.

Actually, to the author's knowledge, very few publications can be found regarding the performance of Rayleigh based OFDR DOFS under fatigue loading. One of this few cases is an engineering note from LUNA Innovations Incorporated, [20]. In this document, is described an experiment where polyimide coated distributed DOFS 
were instrumented to fiberglass coupons and subjected to a $+/-2000 \mu \varepsilon$ and $+/-4000 \mu \varepsilon$ cyclic load for 1000 cycles each. The fiber sensors were interrogated by their ODiSI B model. The results revealed a superior performance of the distributed optical fiber sensors when compared with resistive gauges since the applied DOFS survived the fatigue tests and demonstrated consistency in their strain measurements through the end of the test in opposition to what was verified on the also instrumented resistive strain gauges' measurements. These later sensors presented displayed cumulative zero-shift in microstrain from an early period of the fatigue test, which just increased in magnitude throughout the test cycle.

Another publication from the manufacturers of the ODiSI system reported on the use of polyimide-coated, low-bend-loss fibers embedded in four layers of a carbon fiber spar cap and surface of a $9 \mathrm{~m} \mathrm{CX}-100$ wind turbine blade [21]. The DOFS were used to detect and monitor intentionally introduced defects in the blade and follow the accumulated damage that resulted from these defects with the blade under fatigue loading until failure. The results of the fiber sensor displayed clear evidence of the increasing strain around the defect locations and were able to predict the failure location. Moreover, the DOFS compared well with also instrumented electric foil gauges.

More recently, Wong et al, 2016 described the use of also a similar Rayleigh based OFDR distributed sensors to monitor the fatigue in a flush step lap joint composite structure used in aerospace engineering [22]. This publication demonstrated that it was possible to monitor the fatigue damage propagation until failure with the used DOFS being also able to follow the crack propagation due to the fatigue loading.

All the previous experiences apply to composite elements, where surface characteristics of the material which may highly affect the fatigue response are 
completely different from the case of concrete elements. Consequently, the authors decided to perform a study to assess the fatigue performance of this technology when applied to reinforced concrete structures. An experimental campaign was devised where a fatigue test was performed on Rayleigh based DOFS instrumented reinforced concrete beams where the load reprised expected real world conditions for the case of highway bridges. As far as the authors know, this is the first time that this subject has been investigated. The devised experimental campaign is introduced and described in the following section.

\section{Experimental test setup}

Two concrete beams were casted, beam FA1 and beam FA2. These beams had identical properties and geometry and were characterized by having a $600 \mathrm{~mm}$ length and a square cross-section of $150 \mathrm{~mm}$ width by $150 \mathrm{~mm}$ height, Figure 1 . As depicted in this figure, the reinforcement of each specimen was constituted by two longitudinal $\phi 12$ rebars and four $\phi 6$ stirrups of S500 grade steel.
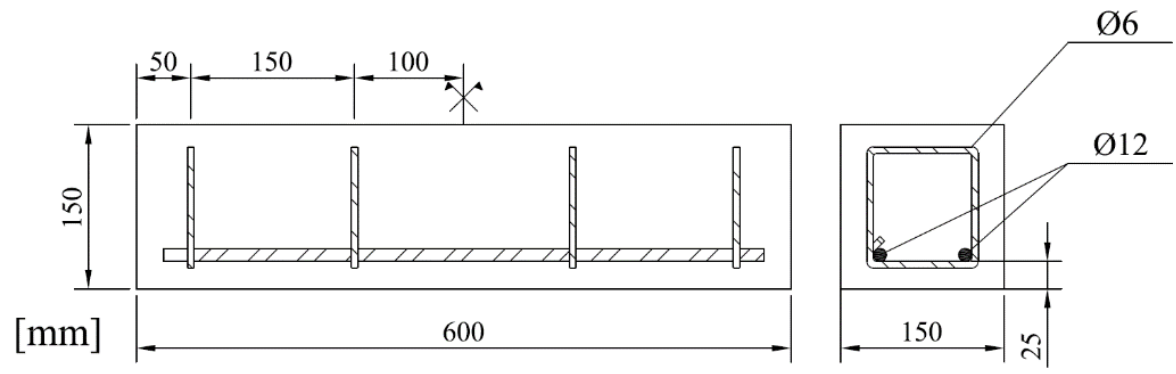

Figure 1. Beam definition scheme (All dimensions in $\mathrm{mm}$ )

In each of the specimens, it was adhered a $5.2 \mathrm{~m}$ long polyimide DOFS performing a pattern with three horizontal segments in its lateral surface and four equal horizontal segments in its bottom surface, Figure 2 and Figure 3. Notwithstanding, for the purposes of this paper, only the collected data from the four bottom surface bonded segments are considered. 
As an additional point of study in this experiment, four different adhesives were used to attach each of the four segments to the bottom surface of the concrete beams, namely neutral cure silicone, polyester, epoxy and cyanoacrylate. This arrangement was the same for both tested beam specimens.

BEAM - BOTTOM VIEW

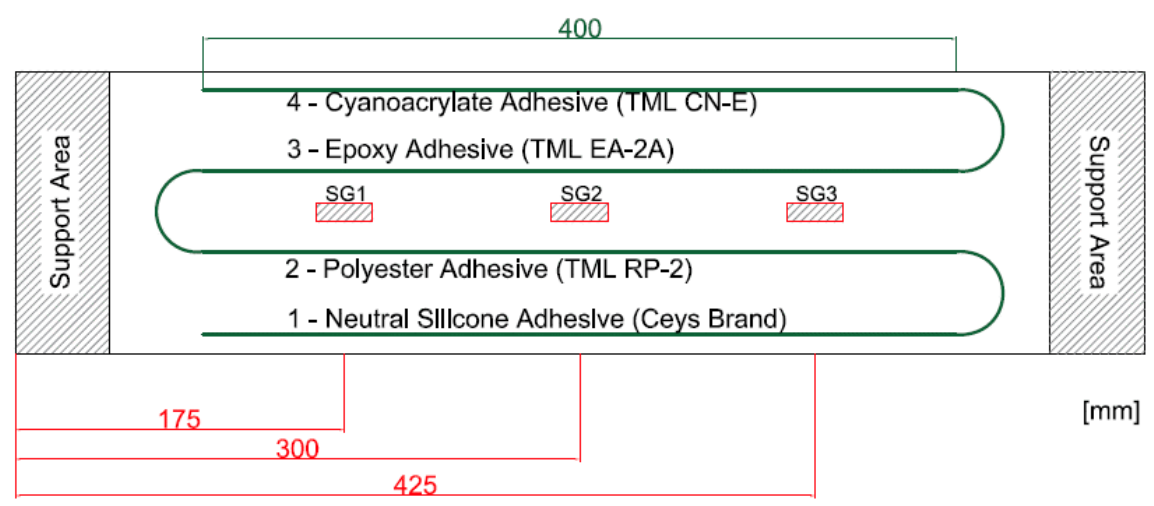

Figure 2. Definition of the sensors attached to the beams and adhesives used

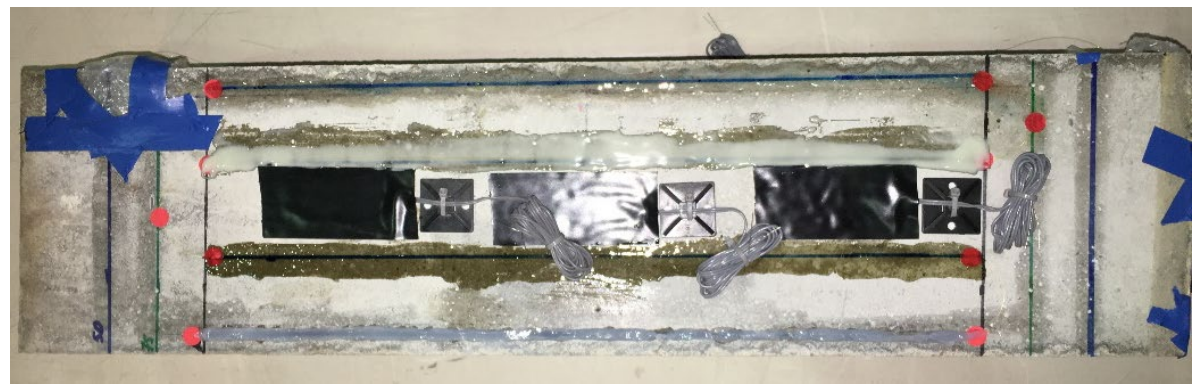

Figure 3. Photograph of the bottom surface of one of the beams with the DOFS and strain gauges

Furthermore, three $30 \mathrm{~mm}$ length electrical strain gauges (SG1, SG2 and SG3) from Tokyo Sokki Kenkyujo Co., Ltd were also attached to the bottom surface of each beam as seen from Figure 2 and Figure 3 for comparison purposes with the DOFS data. As the specimens were loaded using a three-point bend test configuration, the measurements obtained by each segment of the DOFS could be directly compared 
between them and the three strain gauges. A displacement transducer (LVDT) was also deployed at mid-span to follow the deflection of each beam.

At the time of the production of beams FA1 and FA2, in order to obtain the mechanical properties of the concrete, additional cylindrical samples were also produced. These samples were tested close to the date of the load test on the beams. Therefore, the mean compressive strength $\left(f_{c m}\right)$, the mean tensile strength $\left(f_{c t m}\right)$ and the mean Young modulus of the concrete $\left(E_{c}\right)$ were obtained. With this, the expected strain where cracking is expected to occur $\left(\varepsilon_{c t}\right)$ was also obtained, as shown in Table 1. Table 1. Concrete mechanical properties

\begin{tabular}{ccccc}
\hline Properties & $f_{c m}[\mathrm{MPa}]$ & $f_{c t m}[\mathrm{MPa}]$ & $E_{c}[\mathrm{MPa}]$ & $\varepsilon_{c t}[\mu \varepsilon]$ \\
\hline Concrete & 48.027 & 3.944 & 37886.64 & 104.1 \\
\hline
\end{tabular}

As mentioned above, the goal was to subject the specimens to a stress range that would closely replicate the real world conditions observed in a standard highway bridge due to vehicular loads. As a result, for the load input and stress range calculation, the reinforced concrete bridge depicted in Figure 4 was assumed.

a)

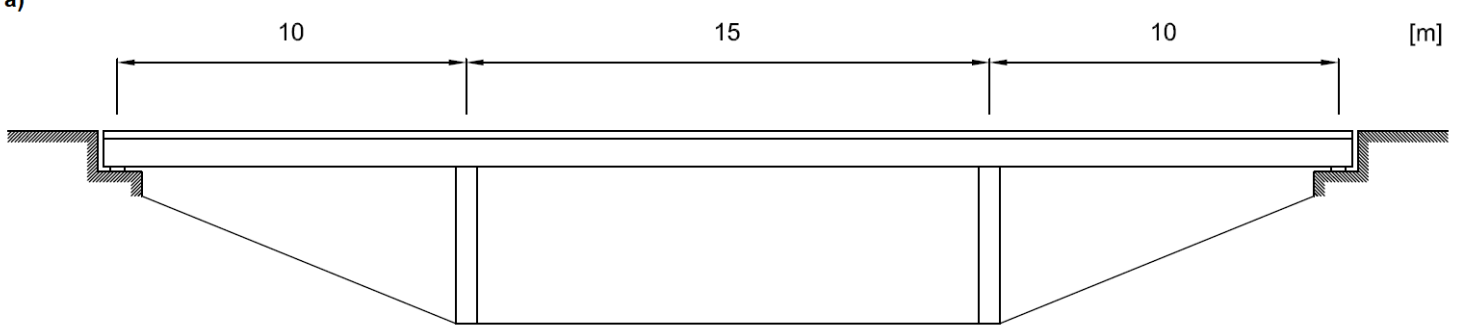

b)

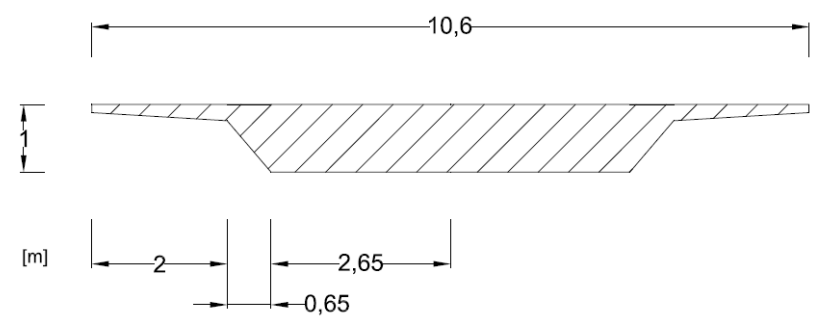

Figure 4. Standard bridge dimensions assumed for the calculation of the load cycles range input - a) elevation; b) cross-section 
In this way, two different load stages were considered for the stress range of the load cycles. The lower stress limit reproduced the sole actuation of the structure's selfweight and permanent loads. The upper stress limit was calculated by adding the traffic load as defined in the live load model to the lower limit. As we were dealing with a fatigue problem, the assumed additional load traffic relevant for fatigue was represented as a four-axle truck with a load of $120 \mathrm{kN}$ by axle and multiplied by a dynamic factor of 1.3 as described in Fatigue Load Model 3 of EN 1991-2 (CEN 2002).[23]

As a result, the considered minimum and maximum load levels and corresponding stress range and the load to be inputted in the fatigue test is described in Table 2.

Table 2. Lower and Upper limits for load cycle range

\begin{tabular}{|c|c|c|c|c|c|}
\hline & $\begin{array}{c}\text { Load } \\
\text { combination }\end{array}$ & $\begin{array}{c}\text { Maximum } \\
\text { bending } \\
\text { moment } \\
{[\mathrm{kN} . \mathrm{m}]}\end{array}$ & $\left.\begin{array}{c}\sigma \\
{[\mathrm{MPa}}\end{array}\right]$ & $\begin{array}{l}\text { Equivalent load } \\
\text { to apply to beam } \\
\text { specimen [kN] }\end{array}$ & $\begin{array}{c}\text { Expected } \\
\text { strain }[\mu \varepsilon]\end{array}$ \\
\hline $\begin{array}{l}\text { Load } \\
\text { Cycle } \\
\text { Level } \\
\text { [inf] }\end{array}$ & permanent load & 3712.9 & 2.612 & 11.75 & 68.9 \\
\hline $\begin{array}{l}\text { Load } \\
\text { Cycle } \\
\text { Level } \\
\text { [sup] }\end{array}$ & $\begin{array}{l}\text { permanent load } \\
+ \text { additional } \\
\text { traffic }\end{array}$ & 4336.3 & 3.050 & 13.73 & 80.5 \\
\hline
\end{tabular}

The cycling load was introduced with a frequency of $4 \mathrm{~Hz}$ and with a sinusoidal profile until reaching 2 million cycles. The frequency was defined such to simulate as much as possible real conditions in concrete bridges when traffic is crossing through. As deduced from tables 1 and 2, the applied load cycles were not expected to generate strains higher than the tensile cracking strain $\varepsilon_{c t}$.

The difference between both beams was that beam FA1 was loaded directly to the 2 million cycles in an un-cracked condition, whereas in beam FA2 the specimen was initially loaded statically until $28 \mathrm{kN}$ (inducing cracking), then unloaded and only afterwards loaded with 2 million cycles identical to beam FA1. The objective was to 
analyse the fatigue performance in both un-cracked and cracked concrete, simulating the cases where the DOFS will be bonded to prestressed (no cracking) or reinforced (cracking) concrete bridges.

A spatial resolution of $1 \mathrm{~cm}$ was selected, what implied an acquisition of strain data from 520 different points along the fiber. It was also defined a sampling acquisition frequency of $0.2 \mathrm{~Hz}$ for the DOFS and $1 \mathrm{~Hz}$ for the strain gauges and load cell information. Finally, due to the extensive duration of the test and the large amount of collected data, the DOFS's measurements were stored for a duration of 5 minutes (1200 load cycles) every 50 thousand cycles.

A photo of each tested beam specimen is depicted in Figure 5.
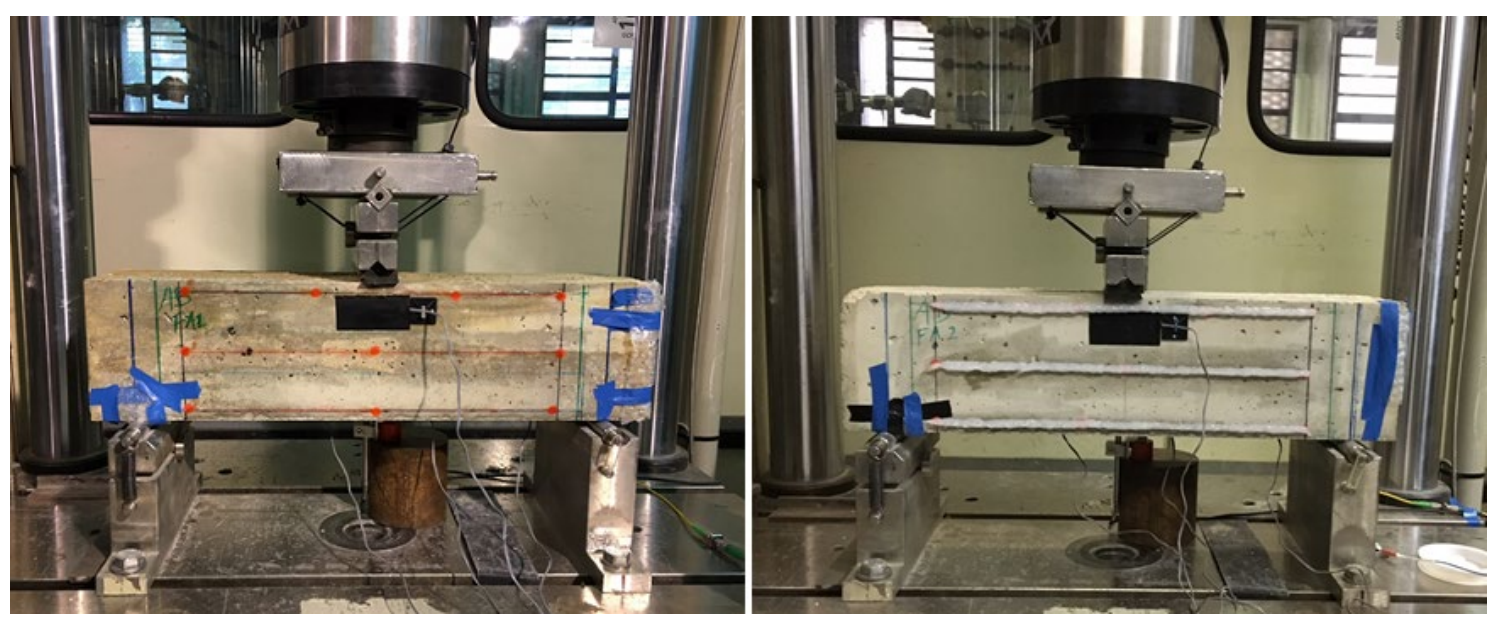

Figure 5. Load arrangement - beam FA1 (left) and beam FA2 (right). The segments of interest for the fatigue analysis are in the bottom surface and cannot be seen in this figure

\section{Discussion of results}

The duration of each fatigue test was around 6 days. Afterwards, the collected data was processed and analysed. The results are presented and discussed in the following sections. 


\subsection{Beam FA1}

When analysing the results of this beam, it is important to mention that during the execution of the load test there was an electrical power shut-off which occurred at the 180664 cycles mark. Afterwards, the test resumed from this point and continued smoothly until it was intentionally stopped shortly after $1,925,000$ cycles. This was due to logistic reasons, which didn't allow for the completion of the total number of 2 million cycles as intended.
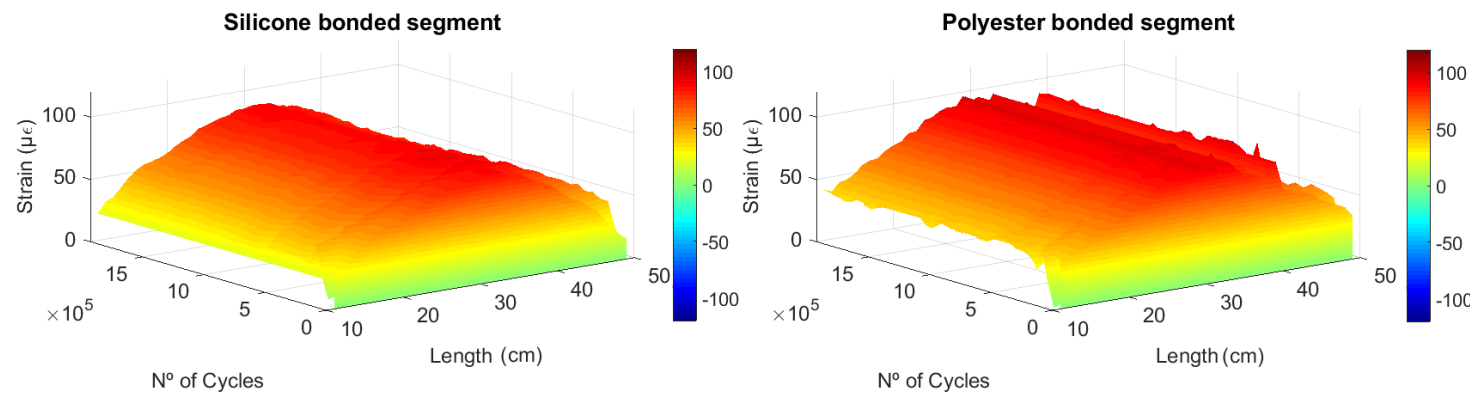

Epoxy bonded segment

Cyanoacrylate bonded segment
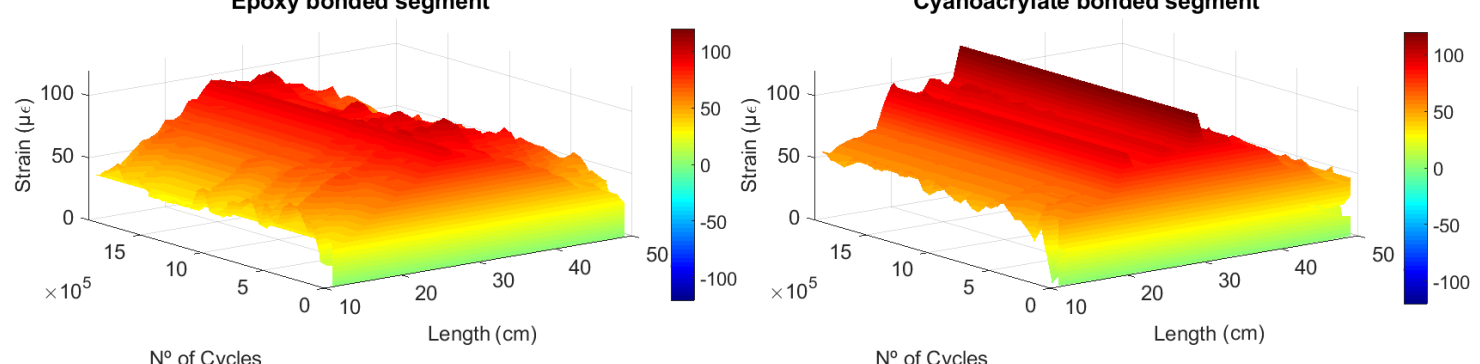

Figure 6. Strain measured by type of bonded segment over the number of load cycles

In Figure 6, it is possible to observe the DOFS' measured strain over the performed number of load cycles and over the bonded length of the DOFS for each adhesive type segment located at the bottom surface of the tested beam. Here it is seen how the different DOFS segments were able to follow the developed strain over the cycling load with different levels of stability. Moreover, it is observed how, in general, the silicone bonded segment presents smoother distributed readings when compared with the other deployed adhesives especially when compared with the cyanoacrylate one. This behaviour was also observed in other tests carried out by the authors [19]. 


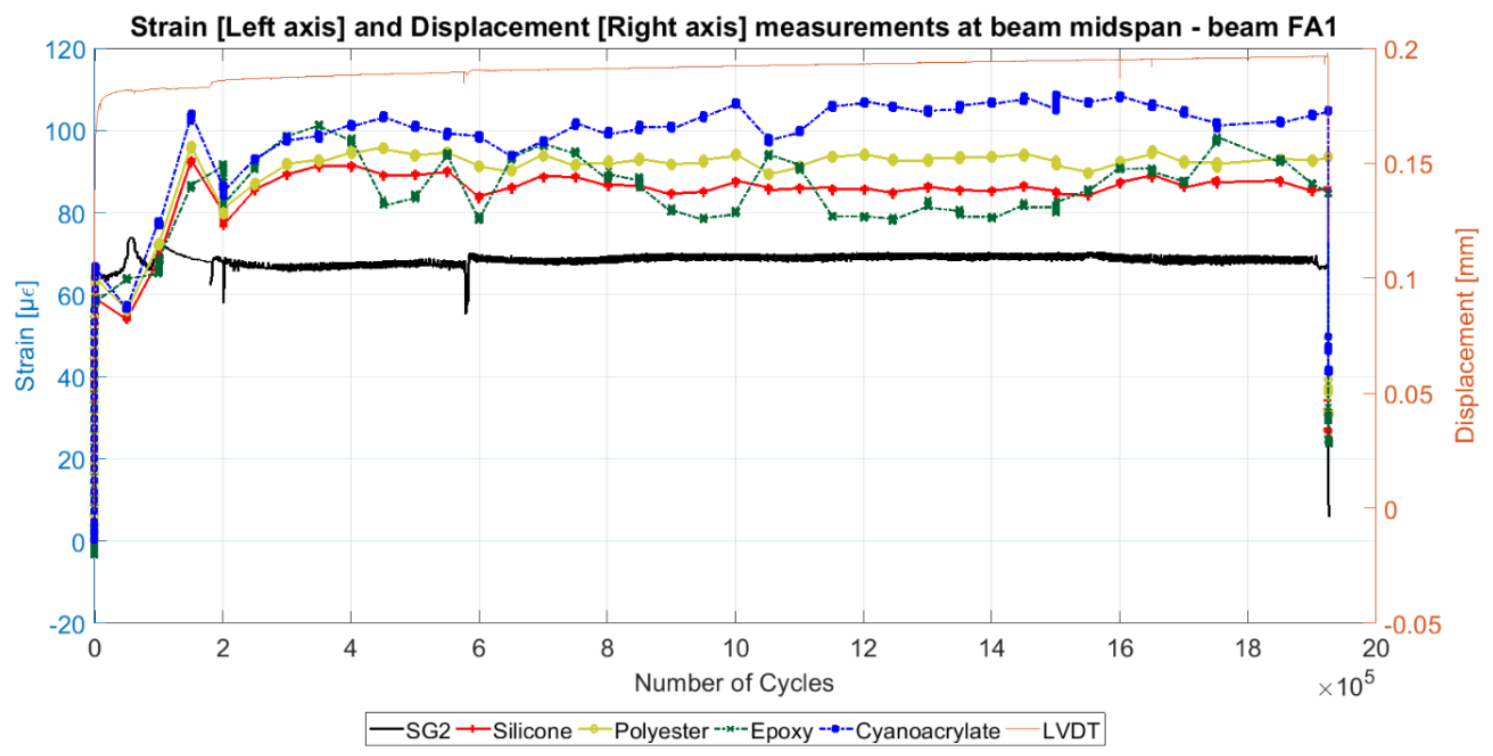

Figure 7. Measured strain and displacement at beam FA1 mid-span over the applied load cycles

Figure 7 summarizes and compares the results obtained by the DOFS and the strain gauges in the centre of the beam, while also plotting the measured displacement at mid-span.

In this figure, it is observed how several interesting events occurred in the first 200k cycles. The measured displacement by the LVDT was progressively increasing, for the same applied load range, from $0.164 \mathrm{~mm}$ to $0.182 \mathrm{~mm}$ in the first $50 \mathrm{k}$ cycles, suggesting an adjustment of beam supports during this period. SG2 and the DOFS' segments also reveal some atypical strain readings during this time period.

It is also observed how all set of sensors seemed to have been affected by the occurrence of the electrical shut off, which occurred around the 180k cycle mark. Close to the $600 \mathrm{k}$ cycles mark it is also seen some atypical readings from SG2, which are then quickly recovered.

Finally and in the context of the goals of the present study, in Figure 7, it is seen how between the $100 \mathrm{k}$ and the $150 \mathrm{k}$ cycle, the readings from the DOFS segments 
diverge substantially from what is observed in the strain gauge without any apparent reason, being then sustained and maintained until the end of the load test.

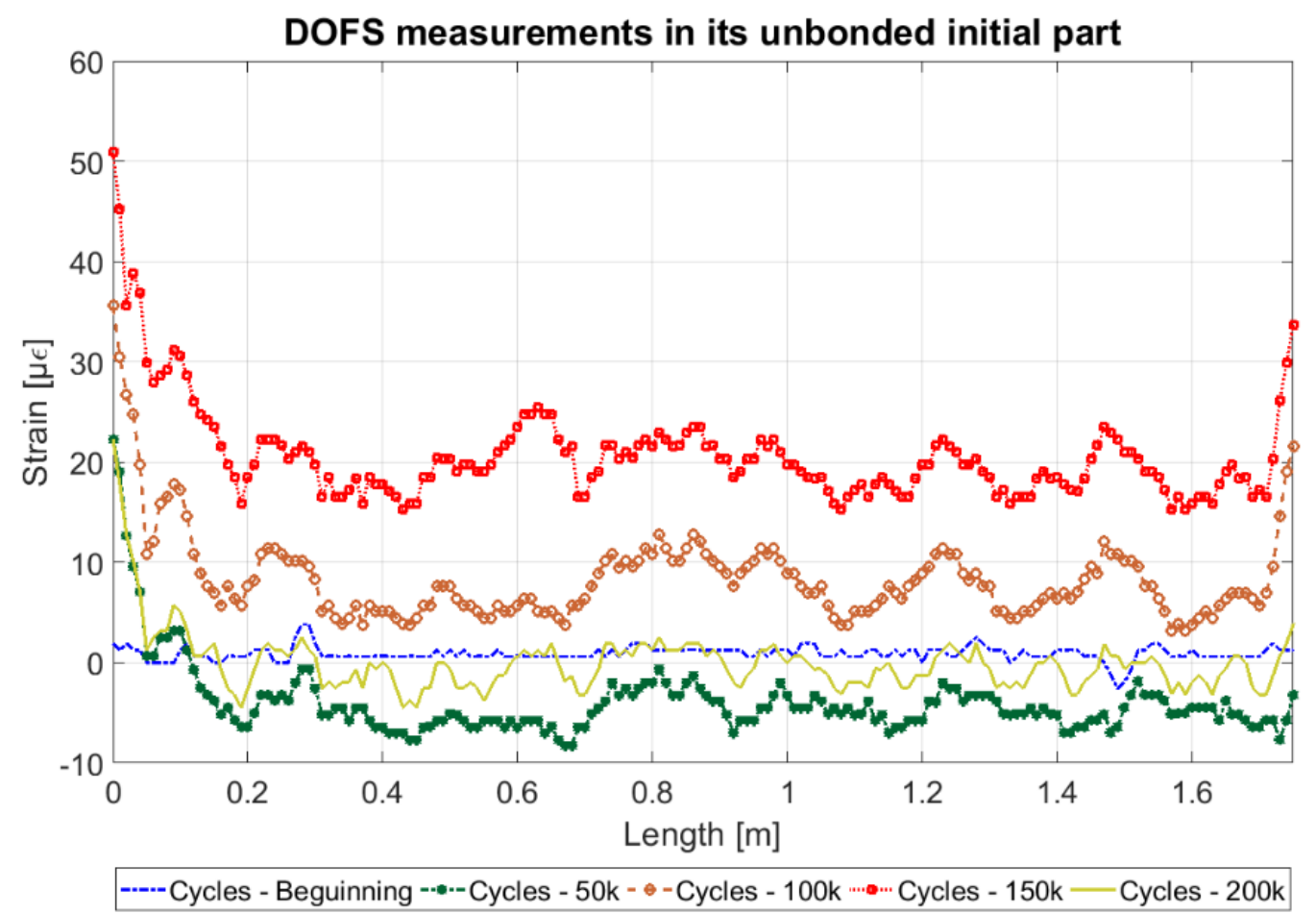

Figure 8. Observed shift of the DOFS measurements observed in its unbonded initial segment

Moreover, this divergence is also observed for the entire length of the deployed DOFS, including the parts of the fiber that are not bonded to the beam, thus discarding any possible influence of a mechanical event. This shift is plotted for the initial unbonded part of the DOFS and for the referred cycles points in Figure 8.

As it is seen in Figure 8, there is a global divergence in the unbonded segment from the first measurements until the $150 \mathrm{k}$ cycle mark. On the next DOFS cycle measurement, after the occurrence of the electrical shut off at $180 \mathrm{k}$, which corresponds to the 200k cycle mark, the aforementioned divergence disappears. However, for the remaining bonded parts of the deployed fiber the new measurements after the $200 \mathrm{k}$ cycle the shift still remains. 
When plotting the longitudinal measured strains by the different bonded DOFS segments at the beginning and end of the applied load cycles jointly with the measurements obtained through the strain gauges, Figure 9 is obtained.
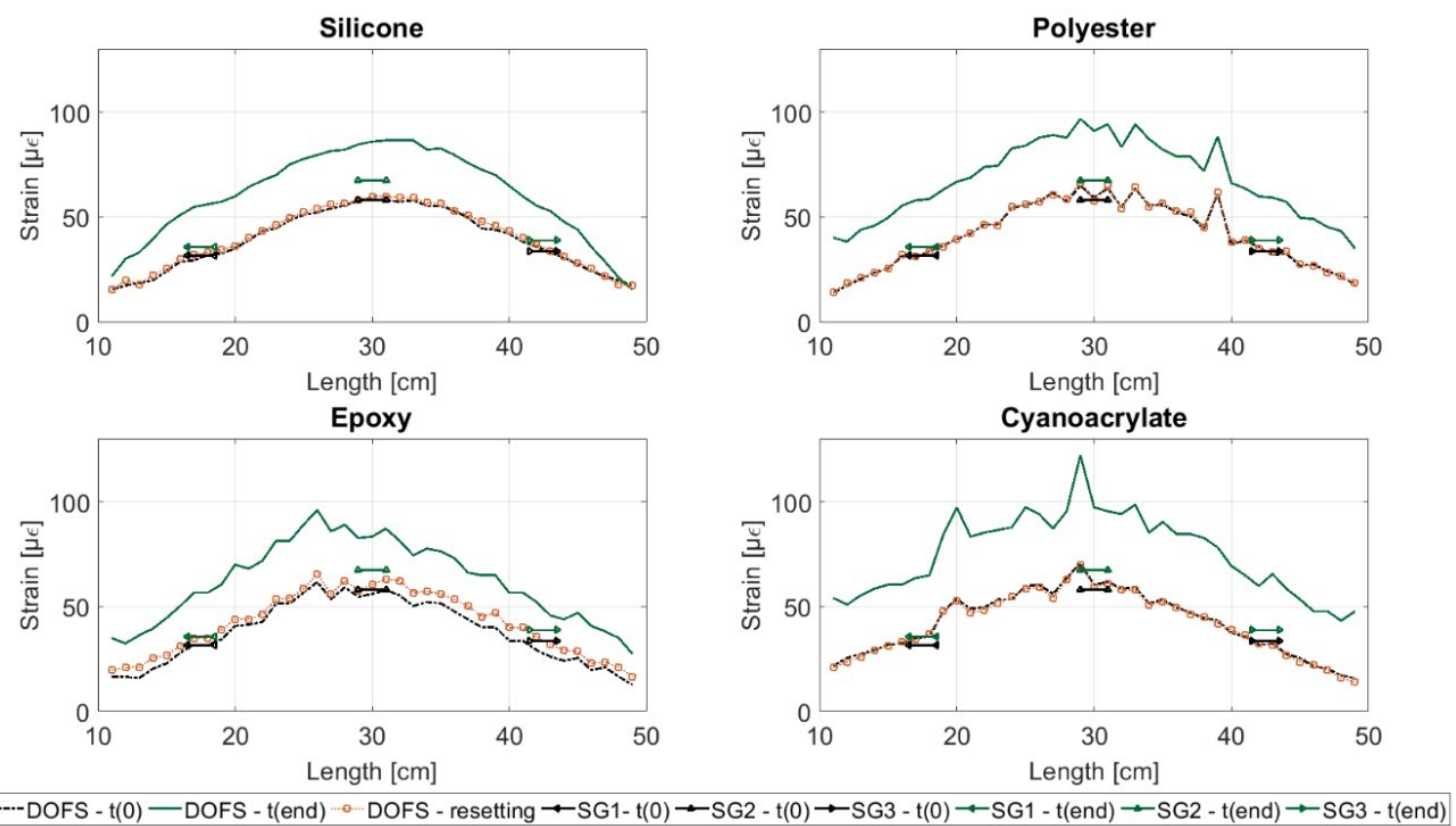

Figure 9. DOFS and strain gauges measurements at the beginning and end of the fatigue test

Here, we can see again how, although both set of sensors agree very well at the beginning of the load cycles, a significant disparity appears at the end. Furthermore, after the end of the load test and the unloading of the specimen (removing of the load representing the permanent state), residual strain measurements remain present in the DOFS. In Figure 9, it is also plotted the result of subtracting this final residual measurement to the readings at the end of the load cycles (DOFS - resetting). In this case, it is observed how the values from the DOFS match almost perfectly the measurements at the beginning of the load cycles and the values from the strain gauges.

The divergence of the measured data observed for the initial unbonded segment of the DOFS before the occurrence of the electrical shut-off at the $180 \mathrm{k}$ cycles mark is 
responsible of the detected difference between the DOFS measurements and the strain gauges.

In this sense, by calculating the average measured values in the initial unbonded segment of the DOFS (between DOFS length coordinate $0.05 \mathrm{~m}$ and $1.60 \mathrm{~m}$ - Figure 8) before the occurrence of the electrical shut-off and subtracting this value to the remaining measured data of the DOFS, the aforementioned shift is eliminated. This is shown in Figure 10, where after the shift correction is possible to notice how the DOFS measurements better follow the strain gauge with appropriate stability along the number of cycles.

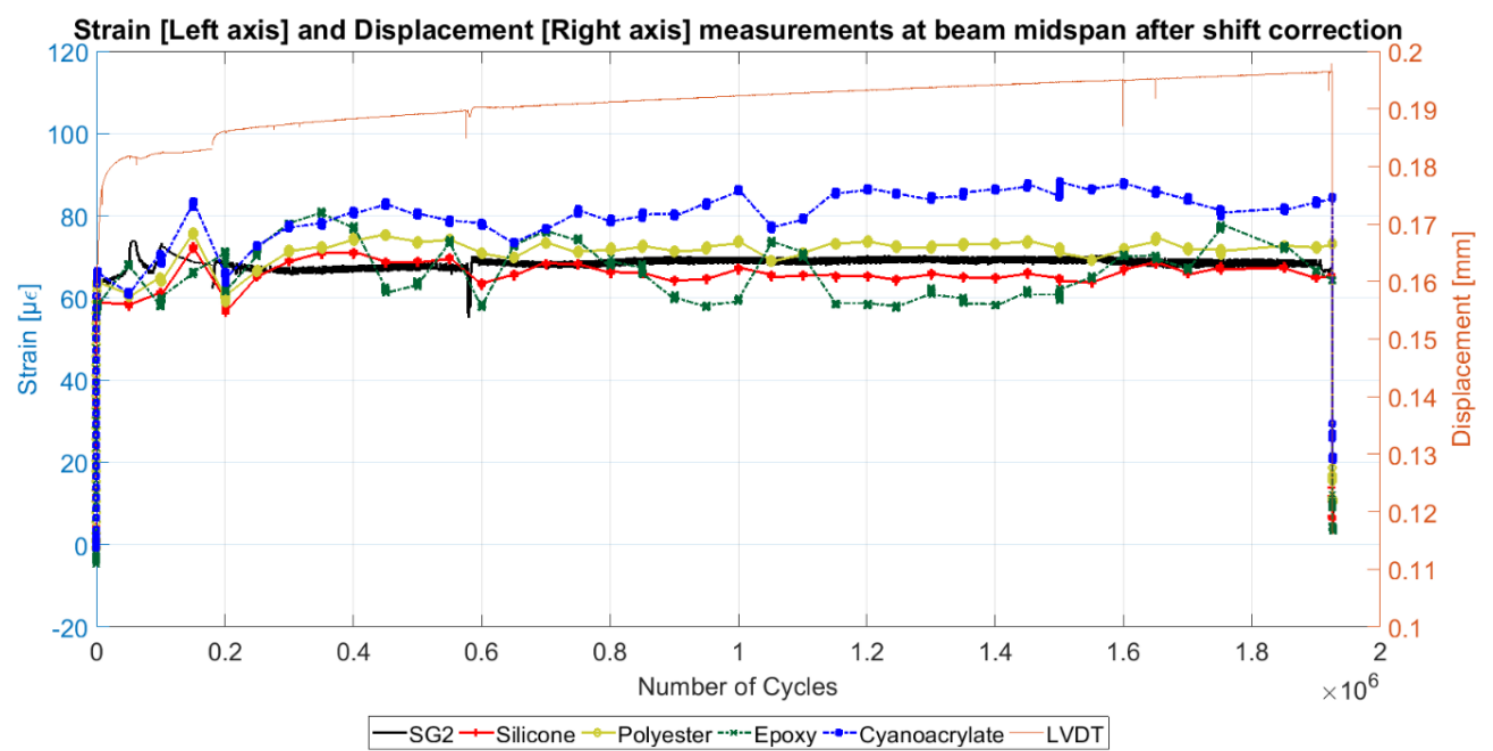

Figure 10. Measured strain and displacement at beam FA1 mid-span over the load cycles after the shift correction

When comparing the results in Figure 10, it is important to remember that the frequency of the cycling load is $4 \mathrm{~Hz}$, while the sampling rate of the acquisition system of the DOFS is of 1 measurement every five seconds and $1 \mathrm{~Hz}$ for the strain gauges. The data was recorded every 50 thousand cycles. Therefore, it was not guaranteed that when recording a result, the strain value obtained in the DOFS coincides at the same point of the sinusoid profile of the load as the strain gauge. A maximum difference in 
the order of magnitude of the applied strain range (around 12 microstrain) is feasible and this is in accordance to what is seen in Figure 10, since the differences between DOFS and strain gauge measurements are somewhat within this range.

\subsection{Beam FA2}

Regarding beam FA2, it was initially and intentionally pre-cracked and only afterwards subjected to the application of two million load cycles with the same load range as beam FA1 and described in Table 2. With this, it was intended to assess the fatigue behaviour of the DOFS measurements when deployed in an existing reinforced concrete structure that may present cracking during its lifetime.

In this way, in Figure 11 is depicted the measured strain in the three strain gauges and the corresponding positions of the different bonded DOFS segments for the precracking stage while also plotting the applied load using the right y-axis. It becomes evident how the measurements in the strain gauges are affected by the cracking.

The corresponding measurements by the DOFS at the same locations as the strain gauges display a general good agreement with them, especially at the beam midpoint, (SG2 location) until cracking is initiated. Cracking is first detected by the cyanoacrylate bonded DOFS segment around minute 15 and then detected by the rest of DOFS segments and SG2 and SG3 at minute 17.5 for a load of $24.67 \mathrm{kN}$. As seen in Figure 12, cracking is located between SG2 and SG3 as detected by the different DOFS bonded segments.

Furthermore, after the cracking detection it is seen in Figure 11 how all DOFS bonded segments and strain gauges measurements follow with more or less agreement the applied load until the beginning of the 2 million load cycles application. However it is observed how after the pre-cracking stage and unloading stage (minute 35 in Figure 11) the strain measurements slightly diverge between the different DOFS segments and 
the strain gauges. This is possibly due to strain redistribution in the element after cracking around the discontinuity that represents the crack and is controlled by the different stiffness of the bonding materials used.

The specific study of these bonding adhesives and their different performance under static loading producing cracking is further detailed and analysed in another publication of the authors [19].
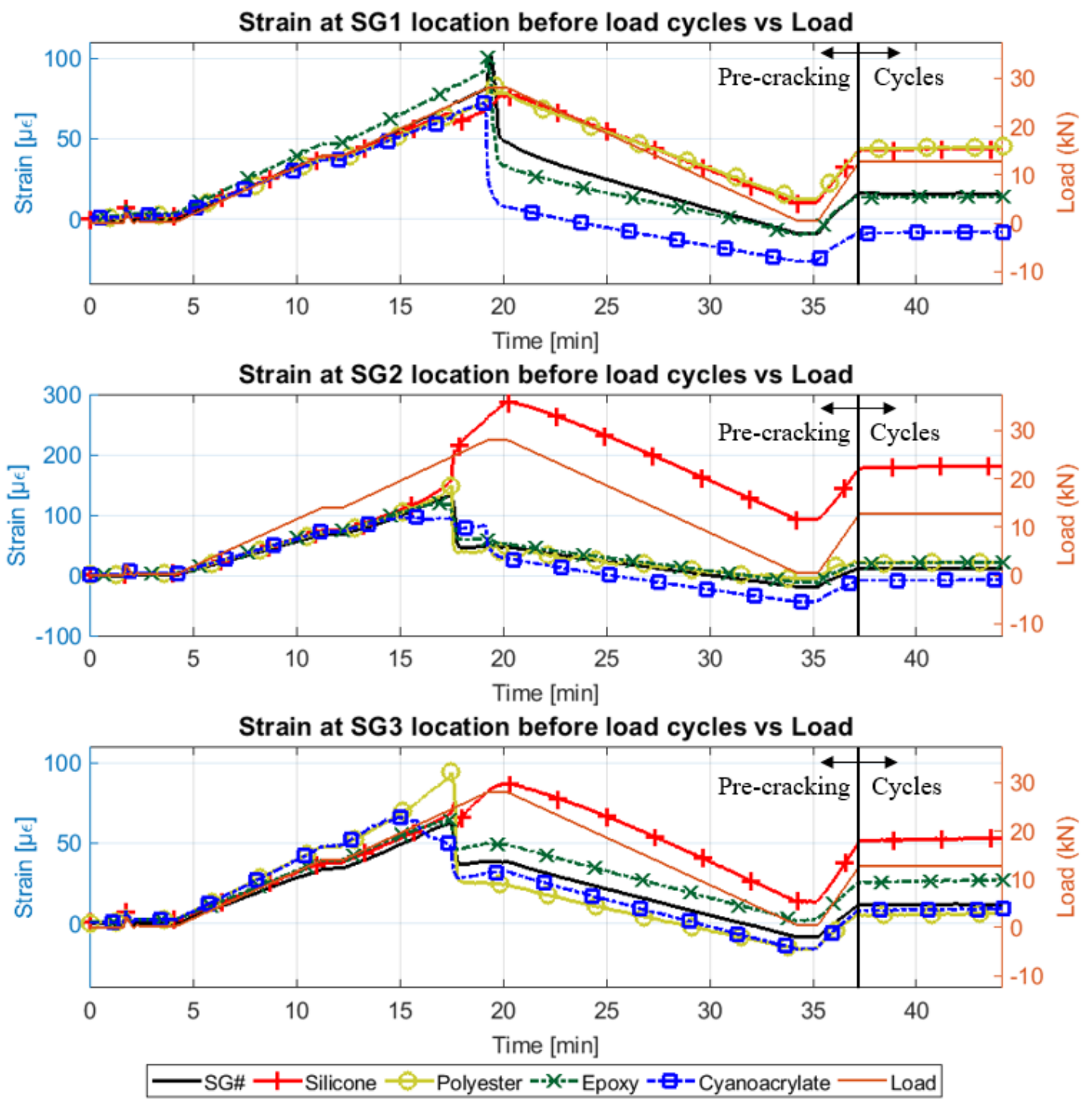

Figure 11. Measured strain and applied load vs time at SG locations in FA2 precracking stage 
In this way, in order to better assess the DOFS performance over the course of the applied number of load cycles, the strain measurements are reset at minute 35 that corresponds to the unloaded stage of the beam after cracking. In Figure 12, due to the scale of the measured strain at the crack location, the measured data of the strain gauges is almost imperceptible; therefore, their location is further highlighted.

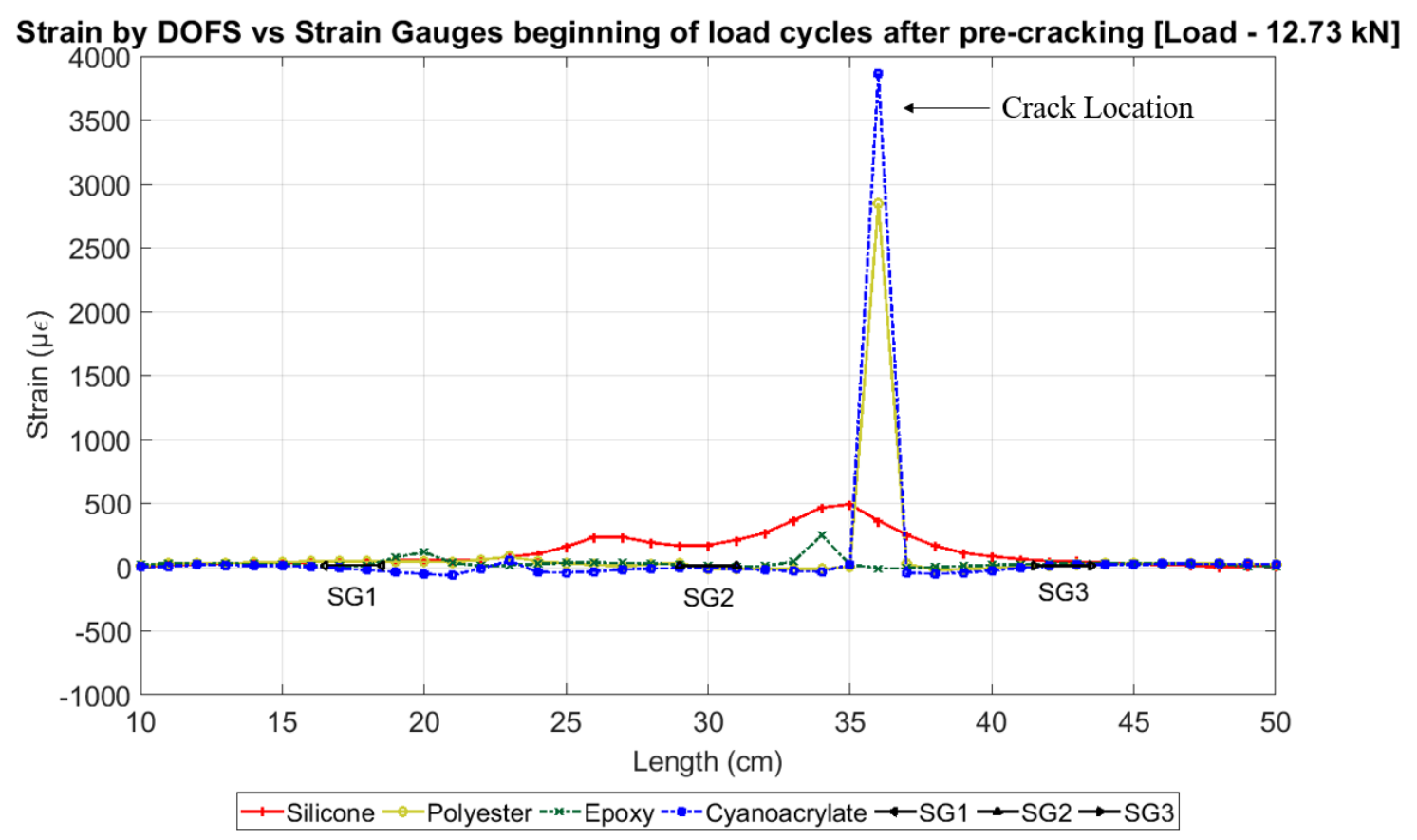

Figure 12. Cracking detection and location at the beginning of the load cycles

Moreover, since as seen in Figure 12, cracking is located between SG2 and SG3, to better compare both sets of strain sensors this is done at the SG1 location, as far as possible, from the cracking location to neglect its influence in the strain measurements. This is shown in Figure 13. 


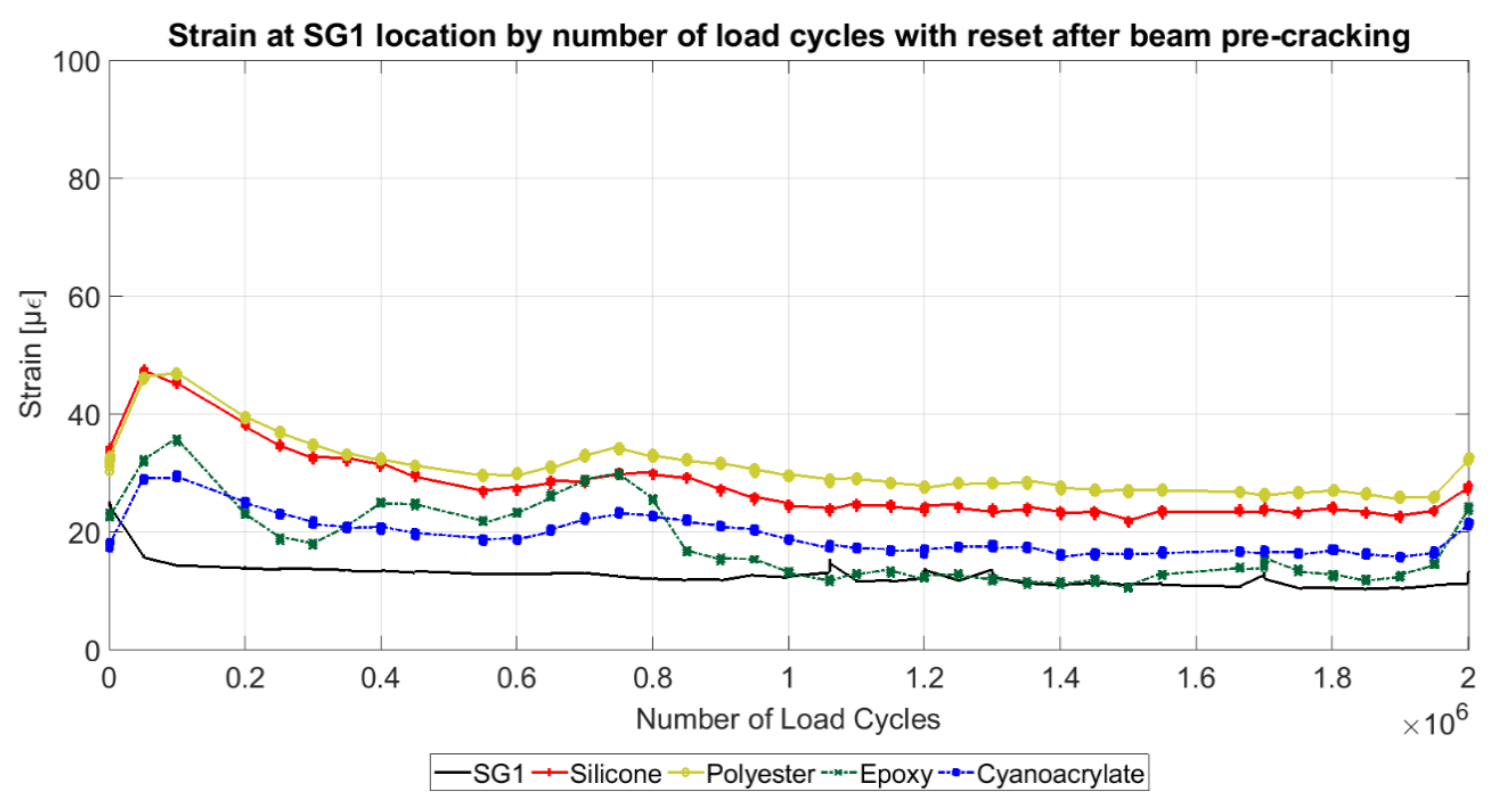

Figure 13. Comparison of strain measurements at SG1 location over load cycles

The DOFS measurements agree reasonably well with the data obtained by the strain gauges. After a small initial variation within the first measured 50k cycles, despite some small fluctuations, all segments present a stabilization of their measurements The observed difference between both set of sensors is more relevant for the DOFS using silicone and polyester and almost negligible for epoxy and cyanoacrylate. These can be justified by these two bonding adhesives lower modulus of elasticity which allows for relative adjustments of the fiber bonded in these two segments influenced by the crack formation.

The remaining observed differences are, as in the case of beam FA1, related with the system strain resolution $( \pm 2 \mu \varepsilon)$, different sampling rates in the acquisition systems and strain range due to the amplitude of the load cycles $(12 \mu \varepsilon)$.

\section{Conclusions}

In this paper, the performance of DOFS when deployed in reinforced concrete elements subjected to fatigue load was assessed. Two reinforced concrete beams (FA1 and FA2) 
were instrumented with a $5.2 \mathrm{~m}$ long polyimide DOFS as well as three strain gauges. Moreover, four different types of adhesives were used to bond the DOFS to the bottom surface of the beams in order to analyse the quality of the corresponding measurements. The beams were subjected to 2 million load cycles with a stress range representative of actual fatigue loading because of traffic in standard highway bridges.

In beam FA1, which was fatigue loaded in un-cracked state, the results show that the DOFS measurements agreed reasonably with what was expected and measured by the strain gauges as well and for all adhesives tested. The measured differences, in the order of magnitude of 12 microstrains are due to the different sampling rates used in the two sets of sensors, the frequency of the load and the resolution of the two types of sensors. However, the results show good stability along the number of cycles and no malfunction due to fatigue effects can be observed.

In beam FA2, which was intentionally pre-cracked before the fatigue test, it was observed how after cracking the DOFS continued to provide strain measurements coherent with the applied load. These values were, however, different depending on the bonding agent used in the DOFS segment and also different of the readings from the strain gauges. In the stage of static load application, before the start of the fatigue test, the best match of the DOFS with the values from the strain gauges is obtained with the epoxy, cyanoacrylate and polyester, whereas the worst is in the DOFS segment bonded with silicone. Afterwards, during the fatigue test, the best performance is obtained for the epoxy and cyanoacrylate adhesives, whose measurements matched perfectly with those from the strain gauges. Larger differences, in the order of 20 microstrains were observed for the other 2 bonding agents. However, in all cases the measurements showed a good stability along the number of cycles, indicating no fatigue failures or debonding in the fibre or the adhesive. 
In this way, this test provided encouraging results regarding the use of this novel technology in real world applications for long-term monitoring periods when a high number of load cycles with low stress range are seen by the monitored concrete structure. The DOFS showed a good performance under fatigue loading, without malfunctions for a number of cycles up to 2 million. The strains measured along the tests were accurate when compared to the results obtained with strain gauges, with good stability and both for the un-cracked and cracked conditions. Therefore, fatigue loading does not affect the performance of the DOFS for obtaining strain profiles along their length. Notwithstanding, it would be interesting in the future to conduct similar tests on larger RC beam specimens and also with higher stress ranges. In order to better compare with the results of strain gauges and to obtain more conclusive results about the accuracy of the strain data, it will be also of interest to carry out tests where the frequency of the cycling load will allow the sampling rate of the DOFS acquisition system to follow the load profile of the variable load.

\section{Acknowledgments}

This project has received funding from the European Union's Horizon 2020 research and innovation programme under the Marie Sklodowska-Curie grant agreement No. 642453.

\section{References}

[1] (American Society of Civil Engineers) ASCE, “2017 Infrastructure Report Card ASCE," 2017.

[2] X. W. Ye, Y. H. Su, and J. P. Han, "Structural Health Monitoring of Civil Infrastructure Using Optical Fiber Sensing Technology: A Comprehensive 
Review.," Sci. World J., vol. 2014, p. 652329, 2014.

[3] B. Glisic, D. Hubbell, D. H. Sigurdardottir, and Y. Yao, "Damage detection and characterization using long-gauge and distributed fiber optic sensors," Opt. Eng., vol. 52, p. 87101, 2013.

[4] J. N. Kudva, C. Marantidis, J. D. Gentry, and E. Blazic, "Smart structures concepts for aircraft structural health monitoring," in 1993 North American Conference on Smart Structures and Materials, 1993, pp. 964-971.

[5] J. M. Lopez-Higuera, L. Rodriguez Cobo, A. Quintela Incera, and A. Cobo, "Fiber Optic Sensors in Structural Health Monitoring," J. Light. Technol., vol. 29, no. 4, pp. 587-608, 2011.

[6] J. R. Casas and P. J. S. Cruz, "Fiber Optic Sensors for Bridge Monitoring," J. Bridg. Eng., vol. 8, no. 6, pp. 362-373, 2003.

[7] J. Sierra-Pérez, M. A. Torres-Arredondo, and A. Güemes, "Damage and nonlinearities detection in wind turbine blades based on strain field pattern recognition. FBGs, OBR and strain gauges comparison,” Compos. Struct., vol. 135, pp. 156-166, Jan. 2016.

[8] P. Ferdinand, "The Evolution of Optical Fiber Sensors Technologies During the 35 Last Years and Their Applications in Structure Health Monitoring," EWSHM7th Eur. Work. Struct. Heal. Monit., 2014.

[9] A. Barrias, J. Casas, and S. Villalba, “A Review of Distributed Optical Fiber Sensors for Civil Engineering Applications," Sensors, vol. 16, no. 5, p. 748, May 2016 
[10] S. Uchida, E. Levenberg, and A. Klar, “On-specimen strain measurement with fiber optic distributed sensing," Measurement, vol. 60, pp. 104-113, 2015.

[11] C.-Y. Hong, Y.-F. Zhang, G.-W. Li, M.-X. Zhang, and Z.-X. Liu, "Recent progress of using Brillouin distributed fiber optic sensors for geotechnical health monitoring," Sensors Actuators A Phys., vol. 258, pp. 131-145, 2017.

[12] R. Regier and N. a. Hoult, "Distributed Strain Behavior of a Reinforced Concrete Bridge: Case Study,” J. Bridg. Eng., vol. 19, no. 12, p. 05014007, 2014.

[13] G. Rodriguez, J. R. . Casas, and S. Villalba, "SHM by DOFS in civil engineering: a review,"Struct. Monit. Maint., vol. 2, no. 4, pp. 357-382, Dec. 2015.

[14] S. Villalba and J. R. Casas, "Application of optical fiber distributed sensing to health monitoring of concrete structures," Mech. Syst. Signal Process., vol. 39, no. 1 , pp. $441-451,2012$.

[15] G. Rodriguez, J. R. Casas, and S. Villalba, "Cracking assessment in concrete structures by distributed optical fiber," Smart Mater. Struct., vol. 24, no. 3, p. 35005,2015

[16] A. Barrias, G. Rodriguez, J. R. Casas, and S. Villalba, “Application of distributed optical fiber sensors for the health monitoring of two real structures in Barcelona,” Struct. Infrastruct. Eng., 2018.

[17] J.-M. Henault, M. Quiertant, S. Delepine-Lesoille, J. Salin, G. Moreau, F. Taillade, and K. Benzarti, "Quantitative strain measurement and crack detection in RC structures using a truly distributed fiber optic sensing system," Constr. Build. Mater., vol. 37, pp. 916-923, Dec. 2012. 
[18] A. Billon, J.-M. Hénault, M. Quiertant, F. Taillade, A. Khadour, R.-P. Martin, and K. Benzarti, "Qualification of a distributed optical fiber sensor bonded to the surface of a concrete structure: a methodology to obtain quantitative strain measurements," Smart Mater. Struct., vol. 24, no. 11, p. 115001, 2015.

[19] A. Barrias, J. R. . Casas, and S. Villalba, "Distributed optical fiber sensors in concrete structures: Performance of bondingadhesives and influence of spatial resolution," Struct. Control Heal. Monit., vol. in press, 2018.

[20] N. A. A. Rahim, M. A. Thoreson, T. Gorney, N. Garg, D. K. Gifford, M. E. Froggatt, and A. K. Sang, "Superior fatigue characteristics of fiber optic strain sensors," LUNA, 2013.

[21] J. R. Pedrazzani, S. M. Klute, D. K. Gifford, A. K. Sang, and M. E. Froggatt, "Embedded and surface mounted fiber optic sensors detect manufacturing defects and accumulated damage as a wind turbine blade is cycled to failure," Luna Innov. Inc, 2012.

[22] L. Wong, N. Chowdhury, J. Wang, W. K. Chiu, and J. Kodikara, "Fatigue damage monitoring of a composite step lap joint using distributed optical fibre sensors," Materials (Basel)., vol. 9, no. 5, p. 374, 2016.

[23] CEN (European Committee For Standardization), “EN 1991-2.” 2002. 